\title{
UTILIZAÇÃO DE FITOTERÁPICOS NO TRATAMENTO DE CONSTIPAÇÃO INTESTINAL
}

\author{
Jaqueline Viol Darroz ${ }^{1}$ \\ Liliane Caldeira Fuso ${ }^{1}$ \\ Natalia Marques Borges ${ }^{1}$ \\ Ana Julia Pereira Santinho Gomes ${ }^{2}$
}

DARROZ, J. V.; FUSO, L. C.; BORGES, N. M.; GOMES, A. J. P. S. Utilização de fitoterápicos no tratamento de constipação intestinal. Arq. Cienc. Saúde UNIPAR, Umuarama, v. 18, n. 2, p, 113-119, maio/ago. 2014.

\begin{abstract}
RESUMO: A incidência da constipação intestinal está frequentemente associada à dieta inadequada e sedentarismo. Acomete indivíduos em qualquer momento da vida. O objetivo deste presente trabalho foi relacionar os medicamentos fitoterápicos atualmente utilizados no tratamento da constipação crônica, apresentando seus mecanismos de ação e seus efeitos adversos. A metodologia empregada baseou-se na pesquisa em base de dados como Scielo, Science Direct, Medline. Os laxantes utilizados clinicamente estão divididos farmacologicamente em 5 tipos, no entanto somente em 2 dessas classes se encontram representantes fitoterápicos: laxativos expansores do bolo fecal e laxativos irritantes ou estimulantes. A utilização de laxantes é produzir evacuações de boa aparência e bem hidratadas, reduzindo os sintomas da constipação crônica.
\end{abstract}

PALAVRAS-CHAVE: Constipação intestinal; Medicamentos fitoterápicos; Plantas medicinais.

\section{PHYTOTHERAPY FOR THE TREATMENT OF CONSTIPATION}

ABSTRACT: The incidence of constipation is frequently associated with an inadequate diet and a sedentary lifestyle. It affects individuals at any time of life. The objective of this study was to relate herbal medicines currently used to treat chronic constipation, presenting its action mechanisms and adverse effects. The methodology used was based on research in databases such as Scielo, Science Direct and Medline. The laxative clinically used are pharmacologically divided into five groups; however, only in two of these groups there are herbal medicine representatives: laxatives that expand the stool and irritant or stimulant laxatives. Laxatives are used to produce good and well-hydrated bowel movements, reducing the symptoms of chronic constipation.

KEYWORDS: Constipation; Phytotherapic drugs; Herbal medicines.

\section{Introdução}

A constipação intestinal é um sintoma muito comum (OLIVEIRA et al., 2005). Sua elevada incidência está frequentemente associada à dieta inadequada, sedentarismo, terapias medicamentosas, alterações endócrinas e metabólicas, além de doenças colônicas, neurológicas, distúrbios psiquiátricos e causas idiopáticas (CRUZ; SUDBRACK; WILSON, 1990).

Acomete indivíduos em qualquer momento da vida, do recém-nascido ao idoso (HARARI et al., 1996; SANT'ANNA; CALÇADO, 1999). Estudos realizados no Brasil mostraram elevada prevalência de constipação em lactentes, pré-escolares e escolares, com valores variando entre $17,5 \%$ e $36,5 \%$ (AGUIRRE et al., 2002; MAFFEI et al., 1997; MOTTA; SILVA, 1998; SANT'ANNA; CALÇADO, 1999; ZASLAVSKY et al., 1988).

A fisiopatologia da constipação é complexa e de etiologia multifatorial. Dentre os fatores etiológicos destacam-se as práticas alimentares, círculo vicioso de evacuação dolorosa, gerando comportamento de retenção fecal, distúrbios da motilidade intestinal e fatores constitucionais e hereditários (LOENING-BAUCKE, 1993; MAFFEI et al., 1994). No entanto, pouco se sabe a respeito da influência dos fatores hereditários na fisiopatologia da constipação, isto é, da relação de concordância de constipação em filhos e seus pais.

Para melhor caracterização, foram estabelecidos critérios que facilitam o diagnóstico, que incluem: ritmo intestinal com menos de três evacuações por semana, sensação de dificuldade para evacuar, fezes pequenas e endurecidas e sensação de evacuação incompleta. Considera-se constipado o paciente que apresentar dois ou mais desses sintomas por período mínimo de três meses ao longo do ano (KAWAGUTI et al., 2008).

A formação de fezes escassas e duras se deve pela diminuição do movimento intestinal, o que origina maior absorção de água secundária pelo tempo de contato mais prolongado com a mucosa intestinal (CARVALHO, 2005).

O tratamento da constipação crônica tem como objetivo aliviar ou eliminar os sintomas já instalados e prevenir ou minimizar a ocorrência de suas complicações (MORAIS; MAFFEI, 2000).

A mudança no estilo de vida, que inclui modificações na dieta, maior prática de atividade física, ingestão de maior quantidade de líquidos, reeducação intestinal e o auxilio de preparados de "fibras vegetais" podem ser os componentes de terapêutica de sucesso para a maioria dos casos de constipação intestinal crônica (SANTOS JUNIOR, 2003).

Os laxantes são medicamentos utilizados no tratamento de constipação crônica. Devem ser usados de maneira parcimoniosa, sempre como auxiliar de tratamento, associados à medidas dietéticas e de reeducação intestinal. $\mathrm{O}$ uso prolongado desses medicamentos pode produzir colite medicamentosa, associada à perda de função intestinal fisiológica. Além disso, podem provocar quadros de diarreia com

DOI: https://doi.org/10.25110/arqsaude.v18i2.2014.5176

1Pós-graduandas do Curso de Especialização em Farmácia Magistral da Universidade do Oeste Paulista, Rua José Bongiovani, 700 Cidade Universitária, CEP 19050-920, Presidente Prudente-SP, Brasil.

${ }^{2}$ Professora Adjunto de Farmacotécnica, Universidade Federal de São João del-Rei, Rua Sebastião Gonçalves Coelho, 400, Bairro Chanadour, CEP 35501296, Divinópolis-MG, Brasil. 
perda excessiva de água e eletrólitos, principalmente potássio, causando fraqueza muscular e astenia. Não devem ser administrados em pacientes com obstrução intestinal, dores abdominais, náuseas ou vômitos (BATISTUZZO; ITAYA; ETO, 2006).

Em comparação ao pequeno número de laxativos sintéticos, o número de laxativos de origem vegetal é vasto (CARVALHO, 2005), sendo assim, várias plantas medicinais oferecem uma fonte alternativa de medicamentos.

De acordo com Santos; Silva (2010) os laxantes utilizados clinicamente estão divididos farmacologicamente em cinco tipos de agentes, como segue: (1) formadores ou expansores do bolo fecal, (2) amolecedores do bolo fecal, (3) lubrificante, (4) osmóticos e (5) estimulantes (secretagogos) ou irritantes do peristaltismo intestinal.

Dentre os fitoterápicos, a classe de metabólitos secundários referente aos heterosídeos antraquinônicos, tem sido usada terapeuticamente com fins laxativos e apresentam-se dentro da classe dos irritantes ou estimulantes do peristaltismo intestinal. Em outra classe denominada expansores do bolo fecal estão presentes as fibras (CARVALHO, 2005).

O objetivo desse trabalho foi relacionar, por meio de uma revisão bibliográfica, os medicamentos fitoterápicos atualmente utilizados no tratamento da constipação crônica, apresentando seus mecanismos de ação e seus efeitos adversos.

\section{Desenvolvimento}

O tratamento medicamentoso da constipação é realizado com a utilização de laxantes. Os laxantes são substâncias que produzem evacuação de boa aparência e bem hidratada (CARVALHO, 2005). Um grande número de pessoas compra e faz uso de laxativos sem procurar um profissional da saúde. Essa classe de medicamentos está entre as de maior procura pela população nas farmácias. Blenkinsopp e Bradley (1996) revelaram que 9 em cada 10 indivíduos tiveram nas duas semanas anteriores ao estudo um problema de saúde, sendo que 3 em cada 4 indivíduos recorreram à automedicação dentre eles $12,8 \%$ recorreram a medicamentos para constipação. No Canadá, em estudo realizado por Segal (1990), numa amostra representativa de alojamentos em Winnipeg e Toronto, verificou-se que 81,6\% dos alojados exerceram automedicação para constipação. Embora somente um terço dos indivíduos afetados procure atendimento médico, os custos de tratamento de constipação chegam à centenas de milhões de dólares por ano despendidos somente com laxativos de venda sem prescrição médica (HIGGINS; JOHANSON, 2004; TALLEY, 2004). A maioria das pessoas desconhece os efeitos colaterais desses insumos farmacêuticos ativos.

Estimativas mostram que o mercado mundial de produtos farmacêuticos movimenta cerca de 320 bilhões de dólares por ano, dos quais 20 bilhões provem de substâncias ativas vegetais. No Brasil, estima-se que $25 \%$ dos 8 bilhões de dólares do faturamento da industria farmacêutica sejam derivados de fitoterápicos (GUERRA; NODARI, 2010). Estes dados revelam o interesse da população mundial por plantas medicinais.

Conforme descrito anteriormente, os laxantes utilizados clinicamente estão divididos farmacologicamente em cinco tipos, no entanto somente em duas dessas classes se encontram representantes fitoterápicos.

Primeiramente, têm-se os laxativos formadores de massa ou incrementadores do bolo fecal, ou seja, aqueles que aumentam a massa intestinal, cujo mecanismo de ação decorre de seu efeito hidrofílico, com acúmulo de líquido no lúmen intestinal, aumento de volume e amolecimento do bolo fecal. O efeito laxativo ocorre entre 12 a 24 horas. É indicada aos pacientes em que não se pode corrigir uma dieta para prisão de ventre. São isentos de efeitos sistêmicos e devem ser administrados com bastante água para se evitar flatulência (SANTOS; SILVA, 2010).

O representante fitoterápico dessa classe é o Psyllium Mill., obtido de sementes das espécies Plantago psyllium e Plantago arenaria (BATISTUZZO; ITAYA; ETO, 2006) que apresenta fibras, compostas por polissacarídeos que por serem ricos em celulose não fracionável pela secreção intestinal e não absorvível, produzem aumento do conteúdo e do peristaltismo intestinal. Outra parte dos polissacarídos é rica em pentoses que facilitam a mobilização e retenção hídrica o que produz fluidificação das fezes (CARVALHO, 2005).

Em adição, este gênero botânico apresenta maior poder de amolecimento das fezes do que o docusato de sódio, por possuir uma maior capacidade de retenção de água, sendo assim mais eficaz em pacientes com constipação crônica, segundo estudo realizado por McRorie e colaboradores (1998), com 170 pacientes com constipação crônica por um período de quatro semanas. Outro estudo demonstrou que as fibras de Psyllium não são fermentadas no cólon intestinal, o que resulta na lubrificação e aumento da expulsão do conteúdo do cólon, além da formação de fezes mais volumosas e úmidas do que aquelas formadas com a utilização de outras fibras (MARLETT; KAJS; FISCHER, 2000).

Essa classe também pode ser utilizada em obesidade, para fornecer ao paciente a sensação de plenitude gástrica, já que aumentam de volume, absorvendo água e formando géis. Deve ser administrado com precaução em pacientes diabéticos, pois reduz a glicemia pós-prandial e altera consequentemente a necessidade de insulina.

Sua dose recomendada é de 5 a 15 g por dia, sendo administrados em envelopes monodoses de $5 \mathrm{~g}$, que devem ser misturados a água, sucos ou leite (BATISTUZZO; ITAYA; ETO, 2006). Podem ser encontrados em especialidades farmacêuticas, como Metamucil ${ }^{\circledR}$ e Plantaben ${ }^{\circledR}$.

As reações adversas desse medicamento estão relacionadas à flatulência, que desaparece em poucos dias, e raramente a ocorrência de dores abdominais e diarréia (PLANTABEN, 2009).

Na segunda classe de laxantes representada por fitoterápicos têm-se os laxativos secretagogos ou agentes com efeitos diretos nas células epiteliais nervosas e da musculatura lisa ou agentes irritantes, que estimulam diretamente o plexo mioentérico aumentando a motilidade intestinal (SANTOS; SILVA, 2010). Os representantes fitoterápicos dessa classe podem atuar no intestino delgado, como é o caso do óleo de rícino, ou no intestino grosso, como é o caso dos compostos antraquinônicos (CARVALHO, 2005).

Esse estímulo pode ocorrer mediante do aumento da permeabilidade da membrana celular por ruptura da continuidade celular. Esses fármacos como resultado de seus mecanismos impedem a reabsorção de eletrólitos e de água 
da luz intestinal, e por isso aumentam significativamente o conteúdo hídrico, o que desencadeia intensamente o peristaltismo intestinal (CARVALHO, 2005).

Neste sentido, apresentam-se os mecanismos de ação e os efeitos adversos de diferentes fitoterápicos utilizados no tratamento de constipação intestinal, como segue: (1) óleo de rícino e (2) compostos antraquinônicos, dentre eles babosa, cáscara sagrada, ruibarbo e sene.

\section{Óleo de rícino}

O óleo de rícino é obtido por expressão a frio (processo de extração por prensagem) das sementes de Ricinus communis, planta originária da Índia que tem como seus principais produtores Brasil, China, Índia, Rússia e Tailândia (MOYNA; HEINZEN, 2010), popularmente conhecida no Brasil como rícino, carrapateiro, mamona e bafureira (COSTA, 2002). As sementes de Ricinus communis contem de 45 a $55 \%$ de óleo fixo, cerca de $20 \%$ de substâncias protéicas, como globulinas, albuminas e glicoproteínas (MOYNA; HEINZEN, 2010), além disso, apresenta uma lecitina extremamente tóxica, a ricina, de estrutura glicoprotéica com propriedades de aglutinar eritrócitos. Outro componente tóxico, que ocorre nas folhas, é a ricinina, uma substância alcaloídica. É ainda descrito no bagaço das sementes (MOYNA; HEINZEN, 2010), a presença de glicoproteínas de ação alergizante em pessoas hipersensíveis (SCHENKEL et al., 2010).

A atividade laxante é atribuída ao ácido ricinoleico, pela propriedade que possui em destacar das paredes do intestino uma camada protetora de lecitinas. No intestino delgado as lipases pancreáticas hidrolisam o óleo a glicerol e ácido ricinoleico, que é um forte surfactante aniônico, favorecendo o acúmulo de água e eletrólitos na luz intestinal e estimulando o peristaltismo (SANTOS; SILVA, 2010).

No caso de deficiência biliar, o óleo de rícino não fica convenientemente emulsionado e a lipase pode não ativá-lo de modo eficaz, e, portanto, deixa de manifestar suas propriedades laxativas (COSTA, 2002). Possui ação laxativa suave e segura, apresentando efeito após 3 a 4 horas da sua administração, não produzindo nenhuma irritação intestinal, a não ser uma ligeira hiperemia. É utilizado antes de procedimentos radiológicos, sendo administrado misturado a suco de laranja, por exemplo, para se mascarar seu sabor nauseoso (CASAMADA, 1968).

O óleo de rícino não apresenta ricina, porém quando preparados por leigos, pode estar contaminado com essa substância, se tornando extremamente tóxico. Os casos mais frequentes de intoxicação ocorrem em crianças pela ingestão das sementes (SCHENKEL et al., 2010).

Comercialmente, o óleo de rícino está presente no Laxol $^{\circledR}$ (LAXOL, 2009) em apresentação de frasco com 60 $\mathrm{mL}$ de óleo de rícino em uma concentração de 99,56 mL de óleo de rícino para cada $100 \mathrm{~mL}$ de solução. A posologia recomendada é de $15 \mathrm{~mL}$, o que promove evacuação aquosa entre 1 a 3 horas, demonstrando sua ação rápida. Em grandes doses pode causar náuseas, vômitos, cólicas e severo efeito purgativo, sendo contraindicado nos casos de obstrução intestinal crônica, doença de Crohn, colite ulcerativa e qualquer outro episódio de inflamação no intestino.

\section{Compostos antraquinônicos}

As antraquinonas podem ser também chamadas de antranóides, derivados antracênicos ou hidroxiantracênicos (FALKENBERG, 2010). Esses compostos derivam do antraceno, núcleo formado por três anéis benzênicos condensados de forma linear (CARVALHO, 2005). A atividade laxante é a responsável pela utilização terapêutica da maioria das plantas que contem quinonas, sendo as substâncias ativas, no caso, os hidroxiantracênicos. As plantas mais utilizadas pela indústria farmacêutica no Brasil e em outros países são: Babosa (Aloe vera), Cáscara Sagrada (Rhamnus purshiana), Ruibarbo (Rheum palmatum) e Sene (Cássia angustifolia).

As antraquinonas, pró-drogas, chegam praticamente intactas ao intestino grosso, onde são digeridas pelas bactérias deste ambiente, perdendo um radical glicídico sendo reduzidas e liberando as formas ativas que são razoavelmente bem absorvidas (SANTOS; SILVA, 2010).

Atualmente, são conhecidos pelo menos três mecanismos que explicam a atividade laxante dos antranóides, como segue: (1) Estimulação direta da contração da musculatura lisa do intestino, aumentando a motilidade intestinal. Este mecanismo está relacionado com a liberação ou com o aumento da síntese de histamina ou outros mediadores, (2) Inibição da reabsorção de água por meio da inativação da bomba de $\mathrm{Na}+/ \mathrm{K}+$ ATPase. Essa bomba parece ser inibida apenas por reina, frângula-emodina, pelas correspondentes antronas e por outras antraquinonas com um grupamento adicional de hidroxila fenólica e (3) Inibição dos canais de cloro, comprovados por inúmeros 1,8-hidroxi-antranoides (antraquinonas e antronas) sendo mais intensa para aloe-emodina (FALKENBERG, 2010).

O consumo regular, por pelo menos um ano, leva frequentemente ao escurecimento da mucosa intestinal do reto e cólon, reversível com a interrupção do uso. Essa alteração pode ser utilizada para diagnóstico do consumo abusivo de laxantes.

Os efeitos adversos e tóxicos da drogas laxantes com derivados antracênicos podem envolver alterações morfológicas no reto e cólon, tais como fissuras anais, prolapsos hemorroidais e outras alterações que não regridem espontaneamente, exigindo intervenção cirúrgica. Também podem ocorrer processos inflamatórios e degenerativos, com risco de redução severa do peristaltismo, o que pode conduzir a atonia. A redução do tônus intestinal leva frequentemente ao uso crônico e abusivo de laxantes, instituindo-se, assim, um círculo vicioso. Outro risco sério associado ao uso abusivo de laxantes é a perda de eletrólitos. A perda de $\mathrm{K}+$ pode levar a redução do tônus intestinal, distúrbios renais, sintomas neuromusculares e distúrbios da formação e condução dos estímulos em nível do miocárdio; especialmente crítico para pacientes usando medicamentos digitálicos no tratamento da insuficiência cardíaca (FALKENBERG, 2010).

\section{Babosa}

A Babosa, também conhecida como Aloe vera tem sido utilizada terapeuticamente desde o período de dominação romana (REYNOLDS; DWECK, 1999; TURNER et al., 2004) e também pelos chineses no tratamento da constipação intestinal (CHUNG et al., 1996a; TIAN; HUA, 2005) 
e como tônico fortificante do estômago (CHUNG et al., 1996b). Entre as drogas vegetais contendo derivados antracênicos, são as plantas que apresentam maior atividade laxante (FALKENBERG, 2010). Existem no mínimo 250 espécies de Aloe conhecidas (KAWAI et al., 1993), mas somente três ou quatro dessas apresentam propriedades medicinais, sendo a Aloe barbadensis a de maior interesse terapêutico e nutricional (ARAÚJO et al., 2002). Apresenta como principal componente, uma substância de estrutura planar, solúvel em água, denominada aloína (GRINDLAY; REYNOLDS, 1986; ODES; MADAR, 1991) que lhe confere propriedades laxantes, purgativas e antiinflamatórias (LEUNG, 1977). Possui ação laxativa potente, porém lenta, cerca de 15 horas após sua administração sendo dos mais irritantes do grupo dos antracênicos, pois estimula todo o cólon intestinal favorecendo o aparecimento de cólicas (CASAMADA, 1968). Em baixas doses, tem ação tônica e digestiva, já em altas doses, apresenta uma ação flogógena, excitando o peristaltismo intestinal (CASAMADA, 1968).

Sua ação é acompanhada por congestão intensa dos órgãos abdominais, propriedade fisiológica que pode ser aproveitada quando se deseja obter desvios sanguinolentos, como nos casos de congestão cerebral e pulmonar. É contra-indicado em indivíduos propensos a hemorróidas, em mulheres grávidas e em crianças (CASAMADA, 1968). Encontrada em associação nas Pílulas de Erva-de-Bicho compostas Imescard $^{\circledR}$ e Pílulas de Lussen ${ }^{\circledR}$ e nas Pílulas de Vida do Dr.Ross ${ }^{\circledR}$ na dosagem de $10 \mathrm{mg}$. Encontrada para manipulação de cápsulas, em dosagens diárias que podem variar de 10 a $60 \mathrm{mg}$, ou 100 a $300 \mathrm{mg}$ se utilizado o extrato seco de Aloe vera. As tinturas também podem ser utilizadas na quantidade de 1 a 2 mL por dia (BATISTUZZO; ITAYA; ETO, 2006).

\section{Cáscara Sagrada}

É originária da costa oeste dos Estados Unidos, atualmente cultivada em outras regiões e também no Canadá. Essa planta começou a ser utilizada pelos índios da América antes da colonização no século XVI como purgativa e tônica, chegando somente no final do século XIX na Europa, sendo descrita botanicamente pela primeira vez, em 1814 (VIANA, 2007).

Os extratos são obtidos da casca seca de Rhamnus purshiana. Essa casca é removida de forma inteira ou fragmentada, submetida a armazenamento por 1 ano e tratada com ar quente antes do uso (VIANA, 2007; FALKENBERG, 2010) pois a droga vegetal recentemente coletada contem antronas o que pode provocar fortes vômitos e até espasmos nos usuários. A Cáscara Sagrada apresenta teores em torno de $6 \%$ de derivados hidroxiantracênicos, dos quais no mínimo $60 \%$ se devem a presença de casacrosídeos (FALKENBERG, 2010). Essas substâncias em contato com a mucosa intestinal aumentam os movimentos peristálticos por meio de mecanismos ainda não estabelecidos, facilitando a eliminação das fezes (VIANA, 2007).

Os hidroxiantracenos e as antraquinonas livres chegam sem serem absorvidas no intestino grosso onde são hidrolizados e transformados pela ação de enzimas em metabólitos ativos. Estes metabólitos estimulam os plexos submucoso e mioentérico do sistema nervoso entérico causando um aumento do peristaltismo (BARRET, 2012).
Associado a esse mecanismo, ocorre ainda um aumento da secreção de água e eletrólitos para luz intestinal, provavelmente devido a inibição dos mecanismos de transporte do ATP dependente do sódio/potássio ou pela inibição dos canais de cloro (VIANA, 2007). Sua ação laxativa é mais suave que a de outros laxantes e se manifesta entre 6 e 8 horas após a sua administração oral, sendo o laxante de escolha em obesidade, quando o paciente apresentar constipação crônica (BATISTUZZO; ITAYA; ETO, 2006). Seu uso não pode ser prolongado por mais de duas semanas pelo risco de se desenvolver as complicações pela utilização abusiva de agentes antraquinônicos.

Está presente em várias especialidades farmacêuticas, de forma isolada como Cáscara Sagrada (Laboratório Herbarium), em que se apresenta $75 \mathrm{mg}$ de Extrato seco de Rhamnus purshiana, padronizado em no mínimo $16 \%$ de cascarosídeo (CASCARA, 2007) ou em associações como em Eparema ${ }^{\circledR}$ em que se apresenta como extrato mole em uma concentração de $1,70 \mathrm{mg}$ de compostos antraquinônicos totais, entre outras. A dosagem diária usual é de 100 a $500 \mathrm{mg}$ de extrato seco; 0,5 a 2 g de pó, 0,3 a $3 \mathrm{~mL}$ de extrato fluido e 1 a $10 \mathrm{~mL}$ de tintura (BATISTUZZO; ITAYA; ETO, 2006).

\section{Ruibarbo}

Os extratos de Ruibarbo são obtidos dos rizomas secos e das raízes desprovidas do periderma, de Rheum officinale e Rheum palmatum. Originário da China e Tibete é cultivado atualmente em toda Ásia e também na Europa. O Ruibarbo considerado verdadeiro é cultivado em altitudes elevadas, com cerca de seis anos. Já a droga procedente de cultivo é obtido de plantas mais jovens já que o cultivo por um período de 6 anos seria pouco viável economicamente. Contem de 3 a $12 \%$ de derivados antracênicos, dos quais 60 a 80 \% correspondem a glicosídeos de frângula-emodina, reína, aloe-emodina, crisofanol e fesciona. Além disso, também apresenta de 2 a $3 \%$ de flavonoides e teores relativamente altos de ácido oxálico, que podem estar associados aos problemas renais relatados em casos de intoxicação por folhas de ruibarbo. Frequentemente se observam falsificações de ruibarbos com outras espécies de Rheum que apresentam teores bem menores de derivados antracênicos que as outras duas espécies consideradas oficinais na maioria dos países (FALKENBERG, 2010).

Possui ação laxativa suave e lenta, por meio da excitação das contrações intestinais, geralmente sem cólicas (CASAMADA, 1968). Difere dos outros laxativos antraquinônicos por exercer uma ação adstringente, devido a presença de taninos, sendo utilizado também no tratamento de diarréias (BATISTUZZO; ITAYA; ETO, 2006). Algumas vezes produz náuseas e vômito, mas raramente vertigens e erupções penfigoides. Por sua ação congestiva sobre os vasos hemorroidais, é contra-indicado nas hemorroidas. Em altas doses pode causar cefaleia, vertigens, vômitos e debilidade muscular (CASAMADA, 1968).

O ruibarbo pode ser encontrado em associações como Eparema ${ }^{\circledR}$ em uma concentração de 1,5 mg de emodina. Também disponível para manipulação de cápsulas com extrato seco, concentração que varia entre 100 e $500 \mathrm{mg}$ ou pó, com concentração entre 0,5 a 2,0 g. Outra forma de utilização são as tinturas e extrato fluido, em quantidades diárias 
de 2 a $20 \mathrm{~mL}$ e 0,3 a $3 \mathrm{~mL}$, respectivamente (BATISTUZZO; ITAYA; ETO, 2006).

\section{Sene}

O Sene é chamado cientificamente de Senna alexandrina Mill. e apresenta como sinonímias científicas Cassia angustifolia Vahl e Cassia senna L. A primeira é nativa do norte da África, Egito e Sudão, enquanto a segunda é nativa do Egito, mas vem sendo cultivada na Índia. Esses dois nomes foram sininomizados (FALKENBERG, 2010). No Brasil chegou por volta do século XIX trazida pelas mãos dos árabes (ROCHA; ROCHA, 2006). Seu efeito laxativo é responsável pela sua utilização atual. O perfil químico do Sene se caracteriza pela presença de substâncias antraquinônicas livres e combinados: crisofanol, aloe-emodina, antranol, reina, reina-8-glicosídeos, reina diglicosídeo, reina antrona-8-glicosídeo (GRANDI et al., 1989). Além destas substâncias citadas, o sene ainda apresenta mucilagens, resinas, flavonóides como o kempferol, gliosídeos naftalênicos, pinitol, açúcares redutores (CRAVEIRO, 1991).

É um dos fármacos mais conceituados como laxante, por não provocar inflamações secundárias muito comuns quando se utilizam laxantes drásticos. Possui atividade laxativa e catártica. É indicado na constipação por inércia intestinal e em condições que exigem facilidade de defecação como fissuras anais e hemorróidas. O efeito do Sene é obtido entre 8 a 10 horas após a sua administração oral (BATISTUZZO; ITAYA; ETO, 2006), pois ocorre a liberação dos heterosídeos no intestino grosso. Neste local, pela ação enzimática da flora bacteriana, ocorrerá hidrólise, com consequente liberação das aglicanas. Estas que atuarão sobre a mucosa, aumentando o peristaltismo.

A histamina estimula a motilidade intestinal e em estudos recentes tem-se mostrado que o Sene causa irritação e degradação dos mastócitos, promovendo a liberação de histamina (VIEIRA, 1992).

Os extratos são obtidos a partir de folíolos e vagens de Cássia senna e Cassia angustifólia e contém glicosídeos antraquinônicos como os senosídeos A e B. Estes glicosídeos são hidrolisados pelas bactérias intestinais, que liberam as antraquinonas ativas no cólon. Dessa forma, é um laxativo suave que não produz irritação estomacal ou no intestino delgado (FALKENBERG, 2010).

O sene pode ser encontrado em associação em formulações para regulação do funcionamento intestinal, como Naturetti $^{\circledR}$ ou Tamarine ${ }^{\circledR}$ em uma concentração de $400 \mathrm{mg}$ e sob forma de extrato seco isolado, como em Senan ${ }^{\circledR}$ que contem $50 \mathrm{mg}$ de extrato seco padronizado de Senna alexandrina Mill. equivalentes a $20 \mathrm{mg}$ de senosídeos. Pode ainda ser encontrado em farmácias magistrais para manipulação de cápsulas ou sob forma líquida. A dosagem diária de extrato seco varia entre 100 e 300 mg, e do pó, 1,0 a 3,0 g diários. Já as tinturas podem ser usadas diariamente de 5 a $20 \mathrm{~mL}$ e os extratos fluidos, de 1 a 5 mL (BATISTUZZO; ITAYA; ETO, 2006).

\section{Conclusão}

O objetivo da utilização de laxantes é produzir evacuações de boa aparência e bem hidratadas, reduzindo os sintomas da constipação crônica. A automedicação é regra habitual para sua utilização, sendo necessários programas de conscientização do paciente sobre seus efeitos colaterais e a necessidade de mudanças na alimentação e estilo de vida.

\section{Referências}

AGUIRRE, A. N. C. et al. Constipação em lactentes: influência do tipo de aleitamento e da ingestão de fibra alimentar. J Pediatr, v. 78, n. 3, p. 202-208, 2002.

ARAÚJO, P. S. et al. Micropropagação de babosa (Aloe vera-Liliaceae). Biotecnologia, Ciência e

Desenvolvimento, v. 25, p. 54-57. 2000.

BARRET, K. E. Visão geral do sistema gastrintestinal: anatomia funcional e regulação. In: RAFF, H.; LEITZKY, M. Fisiologia médica: uma abordagem integrada. Porto Alegre: AMGH, 2012. p. 491-505.

BATISTUZZO, J. A. O.; ITAYA, M.; ETO, Y. Formulário Médico-Farmacêutico. 3. ed. São Paulo: Pharmabooks, 2006. 670p.

BLENKINSOPP, A.; BRADLEY, C. Patients, society and the increase in self-medication. BMJ, v. 312, p. 629-632, 1996.

CARVALHO, J. C. T. Formulações fitoterápicas para distúrbios do aparelho digestório. In: Carvalho, J.C.T. Formulário Médico-Farmacêutico de Fitoterapia. Alfenas: Ciência Brasilis, 2005. p. 27-33.

CÁSCARA SAGRADA. Responsável Técnico: Anny M. Trentini. Colombo: Herbarium Laboratório Botânico Ltda. 2007. Bula de Remédio. Disponível em: <http.//www. herbarium.net/ herbarium_site/produtos/bulas/cascara sagrada.pdf $>$. Acesso em: 10 jun. 2009.

\section{CASAMADA, S. M. Farmacognosia con}

Farmacodinamia. Barcelona: Editorial Cientifico Medica. 1968.1148p.

CHUNG, J. H. et al. Acceleration of the alcohol oxidation rate in rats with aloin, a quinone derivative of Aloe.

Biochem Pharmacol, v. 52, n. 9, p. 1461-1468, 1996a.

CHUNG, J. H. et al. Effect of aloin, a quinone derivative of aloe, on ethanol metabolism in the rat. Medical Sci Res, v. 24, n. 6, p. 421-423, 1996 b.

COSTA, A. F. Farmacognosia. Lisboa: Fundação Calouste Gulbenkian, 2002. 1031p.

CRAVEIRO, A. A. Constituintes Químicos Ativos de Plantas Brasileiras. Ceará. Edições UFC. 1991. 416p.

CRUZ, J. V.; SUDBRACK, C.; WILSON, T. J. Tratamento cirúrgico da retocele como causa de constipação. Rev Bras Coloproct, v. 10, n. 1, p. 5-7, 1990. 
EPAREMA. Responsável técnico Wagner Moi. Santo Amaro: NYCOMED PHARMA, 2009. Bula de remédio.

FALKENBERG, M. B. Quinonas. In: SIMÕES, C. M. O. (Org.) et al. Farmacognosia: da planta ao medicamento. 6.ed. Porto Alegre: Editora da UFRGS, 2010. p. 657-683.

GRANDI, T. S. M. et al. Plantas Medicinais de Minas Gerais, Brasil. Acta Bot Bras, v. 3, n. 2, p. 185-224, 1989.

GRINDLAY, D.; REYNOLDS, T. The Aloe vera phenomenon: a review of the properties and modern uses of the leaf parenchyma gel. J Ethnopharmacol, v. 16, n. 2-3, p. 117-151, 1986.

GUERRA, M. P.; NODARI, R. O. Biodiversidade: aspectos biológicos, geográficos, legais e éticos. In: SIMÕES, C.M.O. (Org.) et al. Farmacognosia: da planta ao medicamento. 6. ed. Porto Alegre: Editora da UFRGS, 2010. p. 13-28.

HARARI, D. et al. Bowel habit in relation to age and gender. Findings from the National Health Interview Survey and clinical implications. Arch Intern Med, v. 156, n. 3 p. 315-320, 1996.

HIGGINS, P. D.; JOHANSON, J. F. Epidemiology of constipation in North America: a systematic review. Am J Gastroenterol, v. 99, n. 4, p. 750-759, 2004.

KAWAGUTI, F. S. et al. Constipação na gravidez. Rev Bras Coloproctol, v. 28, n. 1, p. 46-49, 2008.

KAWAI, K. et al. Tissue-Culture of Aloe-arborescens Miller var. natalensis Berger. Phytotherapy Res, v. 7, p. S5- S10, 1993.

LAXOL. Responsável Técnico Alberto R.V. Stein. Rio de Janeiro: Laboratório Daudt Oliveira Ltda, 2009. Bula de Remédio.

LEUNG, A.Y. Aloe vera in cosmetics. Drug Cosmet Ind, v. 120, n. 6, p. 34-38, 1977.

LOENING-BAUCKE, V. Chronic constipation in children. Gastroenterology. v. 105, n. 5, p. 1557-1564, 1993.

MAFFEI, H. V. L. et al. História clínica e alimentar de crianças atendidas em ambulatório de gastroenterologia pediática (GEP) com constipação intestinal crônica funcional (CICF) e suas possíveis complicações. J Pediatr, v. 70, n. 5, p. 280-286, 1994.

MAFFEI, H. V. L. et al. Prevalência de constipação intestinal em escolares do ciclo básico. J Pediatr, v. 73, n. 5, p. 340-344, 1997.

MARLETT, J. A.; KAJS, T. M.; FISCHER, M. H. An unfermented gel component of psyllium seed husk promotes laxation as a lubricant in humans. Am J Clin Nutr, v. 72, n. 3, p. 784-789, 2000.
McRORIE, J. W. et al. Psyllium is superior to docusate sodium for treatment of chronic constipation. Aliment Pharmacol Ther, v. 12, n. 5, p. 491-497, 1998.

MORAIS, M. B.; MAFFEI, H. V. L. Constipação intestinal. J Pediatr, v. 76, p. S-147-156, 2000. Suplemento 2.

MOTTA, M. E. F. A.; SILVA, G. A. P. Constipação intestinal crônica funcional na infância: diagnóstico e prevalência em uma comunidade de baixa renda. J Pediatr, v. 74, n. 6, p. 451-454, 1998.

MOYNA, P.; HEINZEN, H. Lípidos: química y productos naturales que los contienen. In: SIMOES, C.M.O. (Org.) et al. Farmacognosia: da Planta ao Medicamento. 6. ed. Porto Alegre: Editora da UFRGS, 2010. p. 435-466.

ODES, H. S.; MADAR, Z. A double-blind trial of a celandin, aloevera and psyllium laxative preparation in adult patients with constipation. Digestion, v. 49, n. 2, p. 65-71, 1991.

OLIVEIRA, S. C. M. et al. Constipação intestinal em mulheres na pós-menopausa. Rev Assoc Med Bras, v. 51, n. 6, p. $334-41,2005$.

PLANTABEN. Responsável Técnico Wagner Moi. Jaguariúna: NYCOMED PHARMA LTDA, 2009. Bula do Remédio.

REYNOLDS, T.; DWECK; A. C. Aloe vera leaf gel: a review update. J Ethnopharmacol, v. 68, n. 1-3, p. 3-37, 1999.

ROCHA, G. M.; ROCHA, M. E. N. Uso popular de plantas medicinais. Saúde \& Ambiente em Revista, v. 1, n. 2, p. 76-85, 2006.

SANT’ANNA, A. M. G. A.; CALÇADO, A. C. Constipation in school-aged children at public schools in Rio de Janeiro, Brazil. J Pediatr Gastroenterol Nutr, v. 29, n. 2 p. 190-193, 1999.

SANTOS, D. R. D.; SILVA, L. R. Farmacologia clínica dos laxantes e antidiarréicos. In: SILVA, P. Farmacologia. 8. ed. Rio de Janeiro: Guanabara Koogan, 2010. p. 888-895.

SANTOS JUNIOR, J. C. M. Laxantes e Purgativos - O Paciente e a Constipação Intestinal. Rev Bras Coloproct, v. 23, n. 2, p. 130-134, 2003.

SEGAL, A. Community Survey of Self-medication Activities. Med Care, v. 28, p. 3010-3310, 1990.

SCHENKEL, E. P. et al. Plantas Tóxicas. In: SIMOES, C.M.O. (Org.) et al. Farmacognosia: da Planta ao Medicamento. 6. ed. Porto Alegre: Editora da UFRGS, 2010. p. 957-993.

TALLEY, N. J. Definitions, epidemiology, and impact of chronic constipation. Rev Gastroenterol Disord, v. 4, p. 
S3-S10, 2004. Suplemento 2.

TIAN, B.; HUA, Y. Concentration-dependence of prooxidant and antioxidant effects of aloin and aloe-emodin on DNA. Food Chemistry, v. 91, n. 3, p. 413-418. 2005.

TURNER, C. E. et al. Evaluation and comparison of commercially available Aloe vera L. products using size exclusion chromatography with refractive index and multi-angle laser light scattering detection. Int Immunopharmacol, v. 4, n. 14, p. 1727-1737, 2004.

VIANA, F. A. C. Estudo comparativo, randomizado para avaliar a eficácia terapêutica da piperazina hexahidratada com extrato fluido de Rhamnus Purshiana no tratamento da ascaridíase. 2007. $156 \mathrm{f}$. Dissertação (Mestrado em Farmacologia) - Departamento de Fisiologia e Farmacologia da Universidade do Ceará, Fortaleza, 2007.

VIERA, L. S. Fitoterapia da Amazônia. Manual de plantas medicinais. A farmácia de Deus. 2.ed. São Paulo: Agronômica Ceres. 1992. 347p.

ZASLAVSKY, C. et al. Constipação intestinal na infância. Um estudo de prevalência. Rev AMRIGS. v. 32, p. 100 $102,1988$. 